\title{
The Neuron as a Controlled Toggle Circuit
}

\author{
John Robert Burger \\ ECE Department, California State University, Northridge, USA \\ Email: jrburger1@gmail.com
}

Received 9 September 2014; revised 8 October 2014; accepted 21 October 2014

Copyright (C) 2014 by author and Scientific Research Publishing Inc.

This work is licensed under the Creative Commons Attribution International License (CC BY). http://creativecommons.org/licenses/by/4.0/

(C) () Open Access

\begin{abstract}
This paper considers a model of a recursive neuron whose circuit the author finds interesting, not because of its financial possibility, but because of its surprising electrical behavior. Below, a recursive neuron is modeled with excitatory and inhibitory triggering, and simulated using Win Spice. This model is shown to be capable of controlled toggling, and so promises energy-efficient, massively parallel computing.
\end{abstract}

\section{Keywords}

Brain Circuits, Neural Circuits, Toggle Circuits, Recursive Neurons

\section{The Recursive Neuron}

Realistic circuit models of neurons with computer analysis began to appear in mid twentieth century [1]. This early work is now settled science, promulgated in all major neuroscience texts. Its wide publication makes it easy and fun to explore idiosyncrasies and obscure implications of the modeling. Some configurations of interest to electrical scientists, such as the recursive neuron, have been brushed over by neuroscientists, who are mainly interested in molecular biology and biochemistry, not circuitry.

The information in this article is not new, except for point of view. A neuron is viewed here as an electrical device, and not just a conveyor of molecules. Realistic neurons are interesting from a circuits and systems perspective and suggest a new direction for circuit theory.

A neuron consists of dendrites, or branches attached to a body, or soma, from which a single axon spreads out to form synapses, or connections to the dendrites of other neurons. Pulses can be triggered in exposed neural membrane, chiefly on dendrites and soma, which are active electrically. Subsequently they propagate along exposed membrane without dispersion with soliton-like properties. Compared to the speed of light, about $3 \times 10^{8}$ $\mathrm{mm} / \mathrm{ms}$, they are slow, roughly $1 \mathrm{~mm} / \mathrm{ms}$, which is not a drawback in a massively parallel biological system.

Should the axon of a neuron feed back to its own dendrite, a single neuron supports a pulse that cycles until stopped by an external force. A cycling pulse would pass by the output port again and again, delivering a pulse 
burst to the port that easily signals other neurons in a brain. Electrically, a neuron is similar to delay line memory, used in erstwhile computers of the last century [2].

It is worth noting that a recursive neuron as in this article is not at all a Hopfield neural network. Such artificial neural networks have to use more than one neuron, and are defined to satisfy stability conditions, implying that the output of a Hopfield neuron is not permitted to feed back to itself. In contrast, the neuron as in this article is required to feed back to itself.

The models below are considered "realistic" as opposed to "artificial". The goal is to rouse experimentation and understanding in neuroscience, as opposed to designing a new system of computer hardware. Notwithstanding the current goal, alternative methods of computing with pulses, as opposed to voltage levels, may come to an engineer's mind, using the models below as a basis. But such imaginings are unintended byproducts.

\section{Environment for Neural Pulses}

Neurons are elongated biological cells with micrometer dimensions, enclosed in a thin 1 to $5 \mathrm{~nm}$ membrane. This membrane has a landscape of transmembrane molecules which, once triggered, form conductive "ion" channels. They subsequently pass sufficient electrical charge to form a substantial, roughly $110 \mathrm{mV}$ amplitude pulse across the membrane. Outside ions such as sodium place positive charge inside the membrane, or otherwise, they draw out negative charge from the interior; concurrently, inside ions such as potassium remove positive charge from the inside so that a definite pulse occurs across membrane capacitance.

In equilibrium, prior to triggering, there is an interior potential of about $-70 \mathrm{mV}$ relative to the outside, because of normal exchanges of charges between the inside and outside ions. This potential seems small, but results in a high field across the thin membrane, roughly $140 \mathrm{kV} / \mathrm{cm}$. Lightening in air requires only about 30 $\mathrm{kV} / \mathrm{cm}$. As a result, while in equilibrium, membrane molecules are held quite tightly.

But if an external force reduces the voltage across the membrane from $-70 \mathrm{mV}$ to about $-55 \mathrm{mV}$, transmembrane molecules begin to relax, and permit positive charge inside. This further triggers transmembrane molecules so that a rising pulse is initiated.

Once channels in the membrane are triggered, sodium ions outside the neuron tend to arrive first, mainly since they are smaller and move faster. The fastest of them might actually plough through the channel to directly capture an electron inside the region. When this happens, the interior contains incorrect ions, which must be pumped out later, a process that consumes calories.

The vast majority of ions in a Maxwell-Boltzmann distribution are moving at relatively low speeds, and are expected to meander near (or into the mouth of) a channel at a very low speed. Electrons inside are free briefly, and like a cloud they tunnel everywhere; outside sodium ions may capture interior electrons, thus contributing to positive charge inside. The net result is a rising voltage inside the neuron.

Potassium ions inside the neuron also approach the ion channels but are larger and slower in average speed. They arrive later, and take over fewer channels. The fastest of them may escape from inside the neuron; but any lost potassium must be replaced, which dissipates calories. The vast majority of potassium ions move slowly near the region of the channel, and some of them may capture an electron from the outside. Actions like this bring negative charge into the region and slow the rise of the voltage pulse slightly.

Positive pulse buildup naturally terminates as positive voltage builds up to a peak of about $+40 \mathrm{mV}$. Near this point a strong reversing field tends to trap ions inside the channel, which are held with a time constant; they repel other ions away from the channel. Meanwhile, the potassium channels keep working, causing the pulse to be reduced in voltage. Eventually it undershoots to about $-90 \mathrm{mV}$, which is below its rest voltage of $-70 \mathrm{mV}$. The associated electrical stress on the molecules supporting the conductive channels causes them to reset, thus clearing and closing all ion channels. In due course, voltage drifts back to equilibrium at $-70 \mathrm{mV}$. Re-triggering is of course possible.

A neural pulse is surely an amazement, and it is an entirely natural process; ideal pulses are relatively efficient because ionic conditions are restored, and voltages have relatively low rates of change, resulting in low charging currents, and low losses. It is not like transistors, in which charge is dissipated in stray resistance during rapid switching, necessitating extra cooling.

\section{Simulation of a Neural Pulse}

Lengths of dendrite can be modeled as in Figure 1 with many segments. Segments could be any convenient 


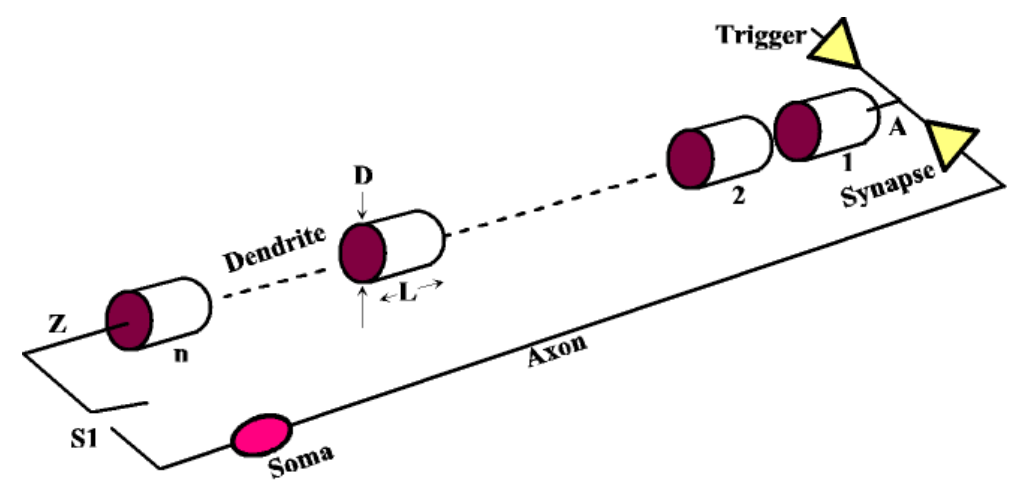

Figure 1. Model of a dendritic path (S1 is a simulation switch to close the loop).

length in a scalable model, but here each segment is assigned a fixed diameter $\mathrm{D}=1 \times 10^{-4} \mathrm{~cm}$, and length $\mathrm{L}=$ $0.1 \mathrm{~cm}$.

Consider a single segment. To facilitate a computerized analysis, realistic parameters are assumed as in Table 1. These parameters are only estimations, but provide a reasonable neural pulse.

Table 2 summarizes equations for computing electrical parameters from physical parameters.

A given segment has the circuit model shown in Figure 2. Series resistance $\mathrm{R}_{1}$ is high, roughly $200 \mathrm{M}$ because the low cross sectional area. Membrane loss $\mathrm{R}_{\mathrm{LOss} 1}$ is fairly high, about $106 \mathrm{M}$, because a membrane is basically extremely thin and is slightly conductive Table 3 lists the parameters used in this model.

This model is piecewise linear in that sodium current is triggered to begin when nodal voltage is $-55 \mathrm{mV}$, but is cut off when voltage reaches $+40 \mathrm{mV}$. Potassium current is also triggered at $-55 \mathrm{mV}$ but is not cut until voltage falls back to $-90 \mathrm{mV}$. These properties were simulated using controlled voltage sources in WinSpice to create hysteresis loops, the outputs of which drive inputs to controlled current sources. Details are published elsewhere [3].

Under this model a pulse appears as in Figure 3. This is reasonably close to what is physically observed experimentally. A pulse in segment 1 soon triggers a pulse in segment 2 when membrane potential reaches $-55 \mathrm{mV}$. Pulses propagate by triggering new identical pulses in resting adjacent segments, appearing to propagate without attenuation or dispersion. After about $15 \mathrm{~ms}$ in this model a pulse appears in segment $\mathrm{n}=10$, which is a distance of $1 \mathrm{~cm}$.

There are several interesting features of such neural pulses. For instance, it is found by simulation that when a pulse reaches an unterminated end of a dendrite, it disappears. But if it encounters a capacitive load of sufficient magnitude, as it would at the central body of a neuron, it is reflected, giving a back propagation. Back propagating pulses sometimes encounter an approaching pulse, and when they collide, both pulses are annihilated. This cuts the rate of arriving pulses in half.

\section{A Natural Toggle Element}

A neuron whose output synapses back to its own input is capable of cycling a single pulse indefinitely, as has been studied by simulation [3]. A single pulse comes from a physically distinctive weak synapse, or alternatively, via a special circuit that converts a pulse burst into a single pulse.

Any given neuron that feeds back on itself can serve as a toggle, delivering either a rest potential, or a stream of regular neural pulses. Combinational logic also could be used to implement a toggle, but at the expense of extra neurons.

Figure 4 shows a method for neural toggling using combinations of excitatory and inhibitory synapses modeled with positive and negative charge injection to model synaptic triggering. This toggle method is perhaps the simplest in that it uses only one neuron. The plan is to excite a circulating pulse at a selected point in the loop, and simultaneously to inhibit pulse propagation at a different point in the loop. Synapses are represented by little amplifiers (triangles). Synapse S1, a weak excitatory synapse, serves to inject a pulse into the loop. Synapse S2, a weak excitatory synapse serves to close the loop so that a single pulse propagates clockwise. The blocks labeled Delay1, 2, 3 and 4 represent the natural delay of a given neuronal path. 
Table 1. Physical parameters.

\begin{tabular}{cc}
\hline Rest Potential & $-70 \mathrm{mV}$ \\
Trigger Voltage (both Na and K currents) & $-55 \mathrm{mV}$ \\
Na Current Cutoff Voltage & $+40 \mathrm{mV}$ \\
K Current Cutoff Voltage & $-90 \mathrm{mV}$ \\
Sodium Current & $67.25 \mathrm{uA} / \mathrm{cm}^{2}$ \\
Potassium Current & $30.4 \mathrm{uA} / \mathrm{cm}^{2}$ \\
Membrane Capacitance & $1 \mathrm{uF} / \mathrm{cm}^{2}$ \\
Membrane Conductance & $0.3 \mathrm{mS} / \mathrm{cm}^{2}$ \\
Internal Resistivity $\rho$ & $15.7 \Omega-\mathrm{cm}^{2}$ \\
\hline
\end{tabular}

Table 2. Equations.

$$
\begin{aligned}
& \mathrm{L}=1 \mathrm{~mm}=0.1 \mathrm{~cm} ; \\
& \mathrm{D}=1 \mathrm{u}=1.0 \mathrm{e}-4 \mathrm{~cm} ; \\
& \mathrm{A}_{\mathrm{CS}}=\pi(\mathrm{D} / 2)^{2} ; \\
& \mathrm{R}_{1}=\rho \mathrm{L} / \mathrm{A}_{\mathrm{CS}}=15.7 \mathrm{~L} / \mathrm{A}_{\mathrm{CS}} \Omega ; \\
& \mathrm{A}_{\mathrm{SIDE}}=\pi \mathrm{DL} ; \\
& \mathrm{C}_{1}=1 \mathrm{uF} / \mathrm{cm}^{2} \mathrm{~A}_{\mathrm{SIDE}} ; \\
& \mathrm{R}_{\mathrm{LOSS} 1}=1 /\left(0.3 \mathrm{e}-3 \mathrm{~A}_{\mathrm{SIDE}}\right) \Omega ; \\
& \mathrm{I}_{\mathrm{Na} 1}=0.06725 \mathrm{~mA} / \mathrm{cm}^{2} \mathrm{~A}_{\mathrm{SIDE}} ; \\
& \mathrm{I}_{\mathrm{K}}=0.0304 \mathrm{~mA} / \mathrm{cm}^{2} \mathrm{~A}_{\mathrm{SIDE}}
\end{aligned}
$$

Table 3. Simulation parameters.

$$
\begin{aligned}
& \mathrm{R}_{1}=200 \mathrm{M} \\
& \mathrm{C}_{1}=31.4 \mathrm{pF} \\
& \mathrm{R}_{\mathrm{LOSS} 1}=106 \mathrm{M} \\
& \mathrm{I}_{\mathrm{Na} 1}=2.11 \mathrm{nA} \\
& \mathrm{I}_{\mathrm{K} 1}=0.955 \mathrm{nA}
\end{aligned}
$$

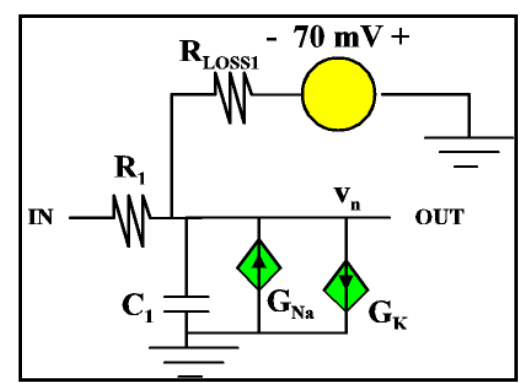

Figure 2. Circuit model of a segment.

Synapses S1, S2 are excitatory synapses modeled with positive charge injection. Synapse S3 is an inhibitory synapse modeled with negative charge injection. The plan is to apply a trigger simultaneously to both synapses S1 and S3 to achieve a toggle neuron.

There are other effective ways to obtain a neural toggle circuit but this one is the simplest. Note that this 


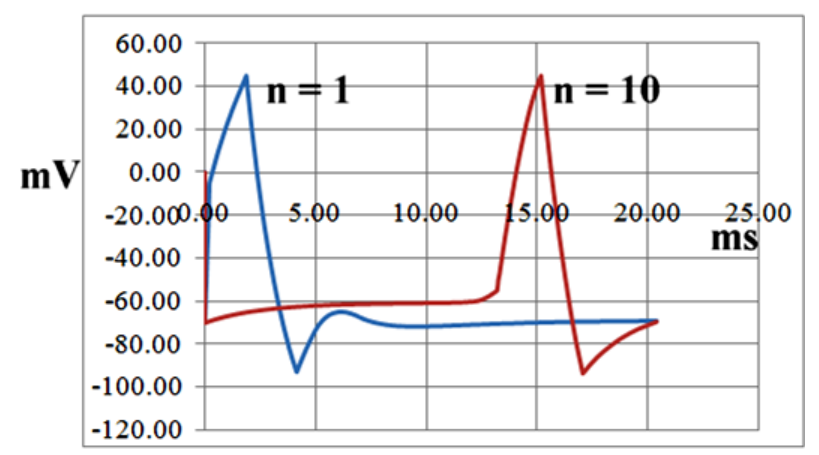

Figure 3. Calculated neural pulse and its propagation from A to Z (S1 open, no feedback).

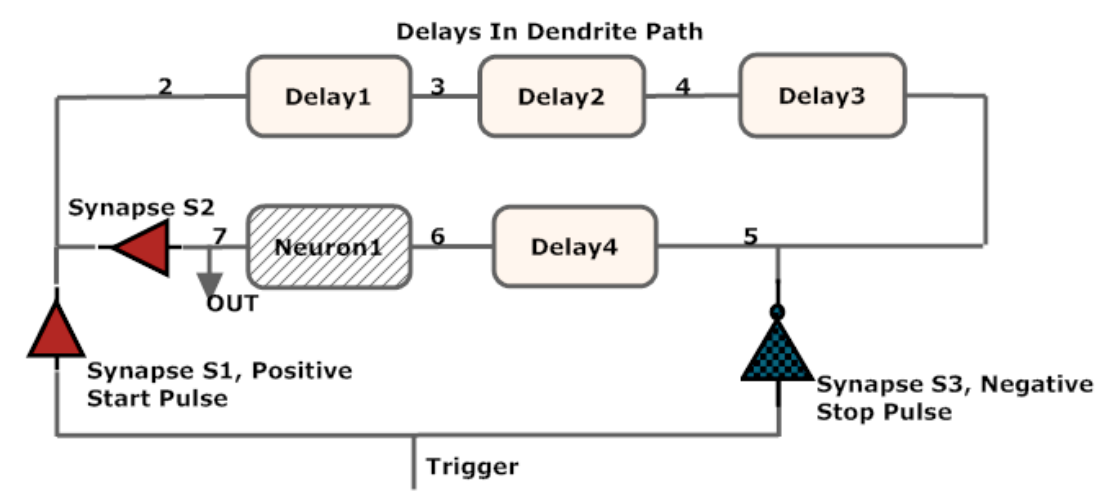

Figure 4. The neuron as a toggle circuit (S1, S2 and S3 are models of synapses).

circuit is easily converted into a set-clear latch, if needed, although clearing is not logically reversible. Toggling is logically reversible with advantages because information is not destroyed. Note that the rate of pulse cycling can be reduced, if desired, by changing the length of the delay path, or alternately by changing ionic conditions to modify delay properties.

Synapse S2, which closes the loop, is modeled with WinSpice code as in Table 4.

This simulates a synapse between a termination on an axon and a receptor on a dendrite. Node 3 is the input; node 2 is the output. The input between nodes 3 and 0 , is referenced to a rest voltage of $-70 \mathrm{mV}$, applied at node 1 in this subcircuit. In addition, a load resistor is used to represent a realistic receptor at the output, and is placed between node 2 and node 1.

\section{Controlled Toggling}

A controlled toggle is one that toggles if and only if one or more control signals are true. A controlled toggle may be implemented as in Figure 5 with the aid of a neural AND gate [3]-[5].

Controlled toggles possess computational possibilities, and may be important to mental processing. In particular, controlled toggling has capability for massively parallel computing, and could serve for the prioritization of mental images and recalls. Logical reversibility and efficiency in controlled toggles are well known advantages [4].

\section{Method of Triggering}

Triggering depends on charging membrane capacitance from rest $(-70 \mathrm{mV})$ up to a threshold at $-55 \mathrm{mV}$. Triggering in this simulation stems from a given source with an amplitude of $1 \mathrm{~V}$ and a pulse width of $0.4 \mathrm{~ms}$ for excitatory, and similarly for inhibitory pulses. Longer triggers are more effective but were unnecessary in this work. Voltage pulses were converted into a nanoampere-range current pulse using a transconductance element.

Positive injection for excitory triggering was created by WinSpice code as in Table 5. 
Table 4. Synapse model (axon to dendrite).

.subckt buf 32
vref $10-70 \mathrm{mV}$
G3 $12315 \mathrm{nA}$
rload $12400 \mathrm{MEG}$
.ends

\section{Table 5. Synapse (positive charge injection).}

.subckt bufv 12

G3 0210 4nA

.ends

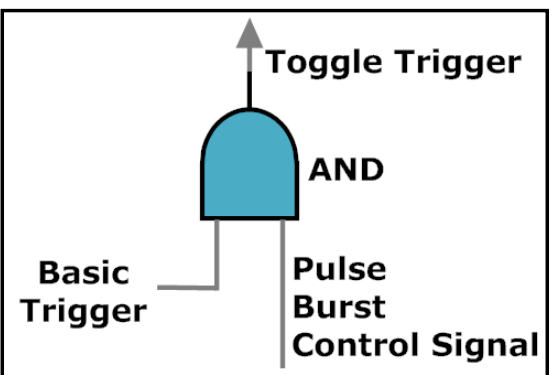

Figure 5. Creating a controlled toggle.

A negative charge injection to node 5 conducts with attenuation to all other nodes and helps to stop cycling pulses no matter where they are. The negative charge injector has WinSpice code as in Table 6.

Since input voltage (Node 1) to this generator is $1 \mathrm{~V}, 0.4 \mathrm{~ms}$, this amounts to $25 \mathrm{nA}, 0.4 \mathrm{~ms}$ from the current source. The pulse is made negative by the order of the outputs at nodes 0,2 which is reversed to 2,0 in the voltage-controlled current source (G3).

The system of triggering in the given toggle element depends on positive charge injection at one point and simultaneous negative charge injection at another well-chosen point in the loop. Pulse cycling will not start if negative injection is applied too close to the positive pulse point. For instance, cycling pulses will not stop in this circuit if negative injection is applied to nodes 3, 4 or 7 in Figure 4, but on and off toggling occurs normally if negative injection is applied to Nodes 5 or 6.

\section{Simulation Results}

A combination of events controls pulses in a recursive neuron. Concerning magnitude of the positive trigger pulse, appropriate triggering was found to occur from roughly $2 \mathrm{nS}$ to $5 \mathrm{nS}$. Below $2 \mathrm{nS}$ a pulse could not be triggered because voltage did not build up to a threshold for triggering; above about $5 \mathrm{nA}$ a single narrow pulse occurred, but would not propagate. This is because the peak at $+40 \mathrm{mV}$ was reached too fast, which did not allow time enough for capacitance in the next segment to charge up to its $-55 \mathrm{mV}$ threshold.

It may be noted that if the negative injection is totally removed from the simulation, this circuit continues to function as a toggle element under certain conditions (Refer to Figure 6).

Here a pulse train (Node 2 in Figure 4, solid blue line) is triggered at zero. Another trigger at about $52 \mathrm{~ms}$ rises up to interfere with the output pulse burst (Node 7 in Figure 4, dashed red line) and prevents further propagation of pulses.

Using only positive charge injection, the delay between triggers has to be within certain limits. For example, toggling worked in this circuit model for trigger separations in the range of roughly 40 to $55 \mathrm{~ms}$ but failed to stop cycling pulses for tested trigger separations below about $40 \mathrm{~ms}$, and for tested trigger separations above about $55 \mathrm{~ms}$. In other words, toggling works only in certain bands of time delays between triggers. 
Table 6. Synapse (negative charge injection).

.subckt bufvN 12

G3 201025 nS

.ends

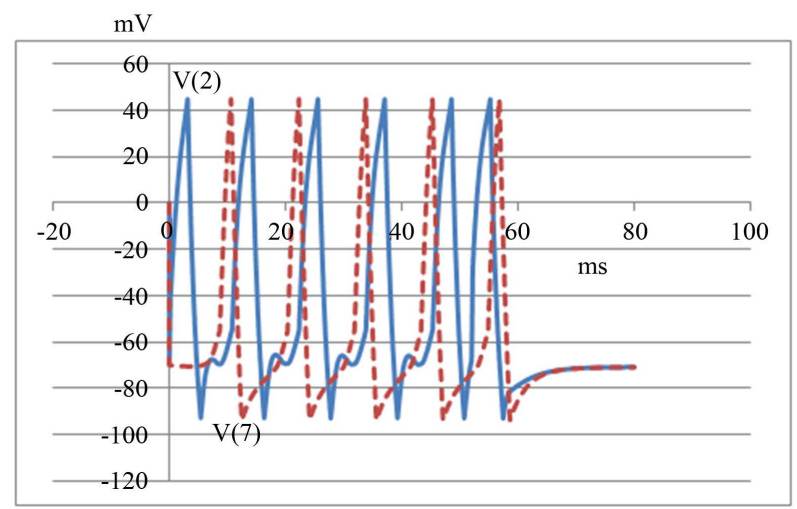

Figure 6. Toggling with positive injection only; triggering at zero and $52 \mathrm{~ms}$.

Toggling to false occurs if a cycling pulse arrives while positive injection is occurring. Voltage is then forced to rise too fast; as a result the pulse then begins to fall before the next segment can charge to a triggering threshold. The net result is that there is not enough charge transferred to the next segment (labeled Delay1 in Figure 4) to cause it to trigger. Pulse cycling thus stops.

When using negative injection (representing an inhibitory synapse), the delays between triggers for toggling are far less critical. Using a $25 \mathrm{nA}$ negative pulse, toggling worked at all tested trigger delays in the range 20 to $80 \mathrm{~ms}$ (Refer to Figure 7).

Here a circulating pulse train is triggered at zero. A significant negative charge is injected after $60 \mathrm{~ms}$ that eventually stops all pulses from cycling, including the original pulse, as well as the new pulse at $60 \mathrm{~ms}$. The solid blue line is the waveform at the point of triggering, Node 1 in Figure 4; the dashed red line is the output pulse burst, Node 7.

This article has been limited to a single neuron working as a toggle element. Clearly there are many neurons in a human brain, about 100 billion $\left(10^{11}\right)$ and even more potential synapses, about 100 trillion $\left(10^{14}\right)$. A large number of other Boolean gates are available in a neuron, some quite novel. Boolean methods are available simply by defining false to be rest, and true to be a pulse stream. An overview for non experts of neural elements, circuits and systems, and how they might support brain operations is readily available [5].

\section{Conclusions}

This article aims to offer a fresh point of view. Decades old simulations of neurons and neural membranes are re-considered from an electrical perspective, as opposed to a chemical perspective. If you are a neuroscientist, you see neural membranes as mainly passing molecules and ions back and forth. This chemical point of view is quite popular but hinders an understanding of the brain as a system.

Understanding a system demands a circuits and systems point of view. Beyond molecules, a brain is obviously a working complexity of circuits. There is no reason to suspect that a brain violates even the smallest aspect of circuit theory or, for that matter, any known principle of physics. So when explaining a brain, circuits and systems theory is without question most relevant.

Circuits engineers generally work with solid state connections in one form or another. The work described below runs against this trend in that it focuses on models of natural circuitry as accomplished by biological neurons. Neural circuits are not new, although they are largely mysterious. Everyday humble neurons hold interest because they have complexity and abilities that range far beyond the transistor. Engineers could learn much from them. 


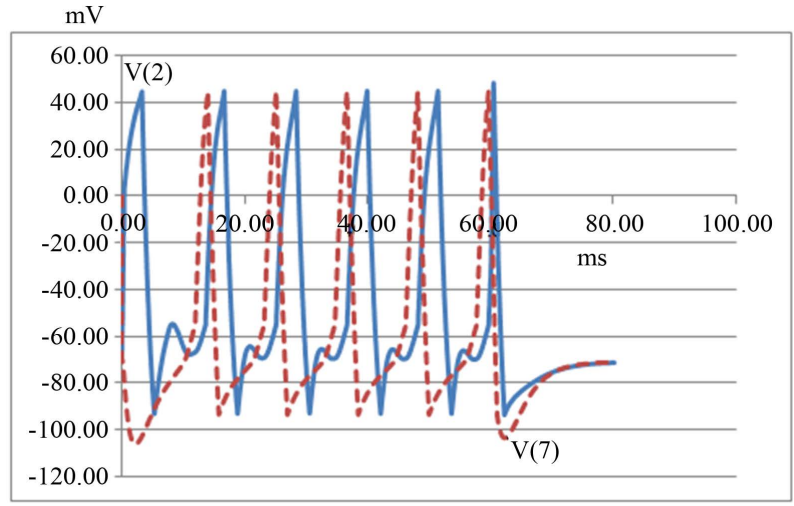

Figure 7. Toggling with positive and negative injection; triggering at zero and $60 \mathrm{~ms}$.

A neural system is quite unlike a solid state system: A neural system depends on voltage pulses, and so it is very different from level sensitive logic. Nevertheless, Boolean logic is relevant, simply by defining true or false to be pulses, or no pulses.

This article has focused on a single element, a toggle element based on a single neuron. A toggle element, when triggered, moves its output from true to false, or from false to true. Triggering is accomplished by a novel combination of positive and negative charge injection. Triggering results in a single pulse that cycles about a loop formed when an axon of a neuron synapses (connects) back to one of its own dendrites. Single pulses are assumed to be available from a weak synapse, or alternately, from a specialized neural circuit.

A simulation is presented above, and it delineates the behavior of a particular recursive neuron working as a toggle switch. Controlled toggles are created by applying an AND gate to the trigger signal. A controlled toggle changes state only if one or more controlling signals are all true. Networks of controlled toggles are known to be capable of massively parallel computing, and may be important for complex brain operations such as memory recall editing.

Controlled toggles are logically reversible, and to some extent may be physically reversible, with implications for energy conservation. Indeed, by avoiding arbitrary resets and clears in which information is lost forever, fewer calories are dissipated during computations.

For example, it has been estimated that a human brain uses only about 6 watts, roughly that of a dim light bulb, and apparently does so without regard to the level of mental effort. Brain operations are important to engineers who might learn from the human brain, famous for ample computing with low power dissipation.

Finally, this article considers a realistic model of a recursive neuron with its promise for energy efficiency and massive computing. The analysis above is the first step toward shedding light on what has been a shadowy area dominated by biologists and neuroscientists who usually do not focus on electrical circuits and systems.

\section{References}

[1] Hodgkin, A.L. and Huxley, A.F. (1952) A Quantitative Description of Membrane Current and Its Application to Conduction and Excitation in Nerve. The Journal of Physiology, 177, 500-544.

[2] Eckert, J.P. (1953) A Survey of Digital Computer Memory Systems. Proc. IRE, October.

[3] Burger, J.R. (2013) Brain Theory from A Circuits and Systems Perspective-How Electrical Science Explains NeuroCircuits, Neuro-Systems, and Qubits. Springer, New York, Appendix A. http://dx.doi.org/10.1007/978-1-4614-6412-9

[4] Burger, J.R. (2009) Human Memory Modeled with Standard Analog and Digital Circuits: Inspiration for Man-Made Computers. John Wiley \& Sons, Hoboken. http://dx.doi.org/10.1002/9780470464250

[5] Burger, J.R. (2014) The Synthesis of a Neural System to Explain Consciousness: Neural Circuits, Neural Systems and Wakefulness for Non-Specialists. Luminare Press, Eugene.

http://www.amazon.com/Synthesis-Neural-System-Explain-Consciousness/dp/1937303284 
Scientific Research Publishing (SCIRP) is one of the largest Open Access journal publishers. It is currently publishing more than 200 open access, online, peer-reviewed journals covering a wide range of academic disciplines. SCIRP serves the worldwide academic communities and contributes to the progress and application of science with its publication.

Other selected journals from SCIRP are listed as below. Submit your manuscript to us via either submit@scirp.org or Online Submission Portal.
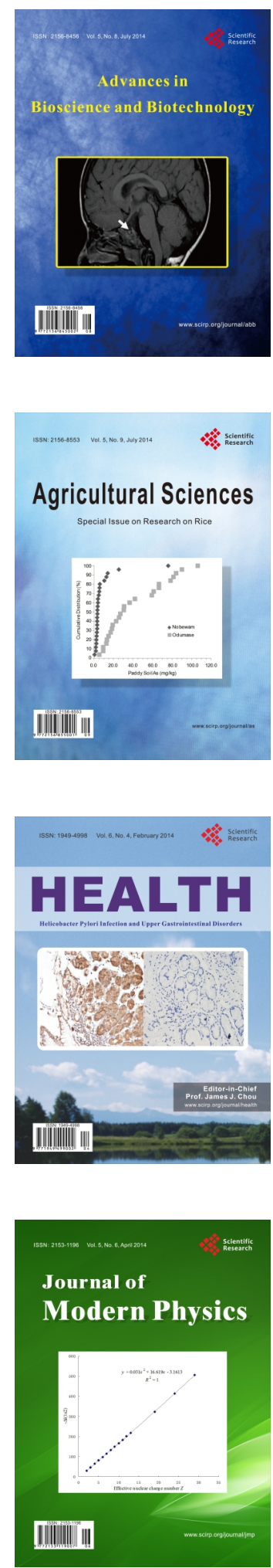
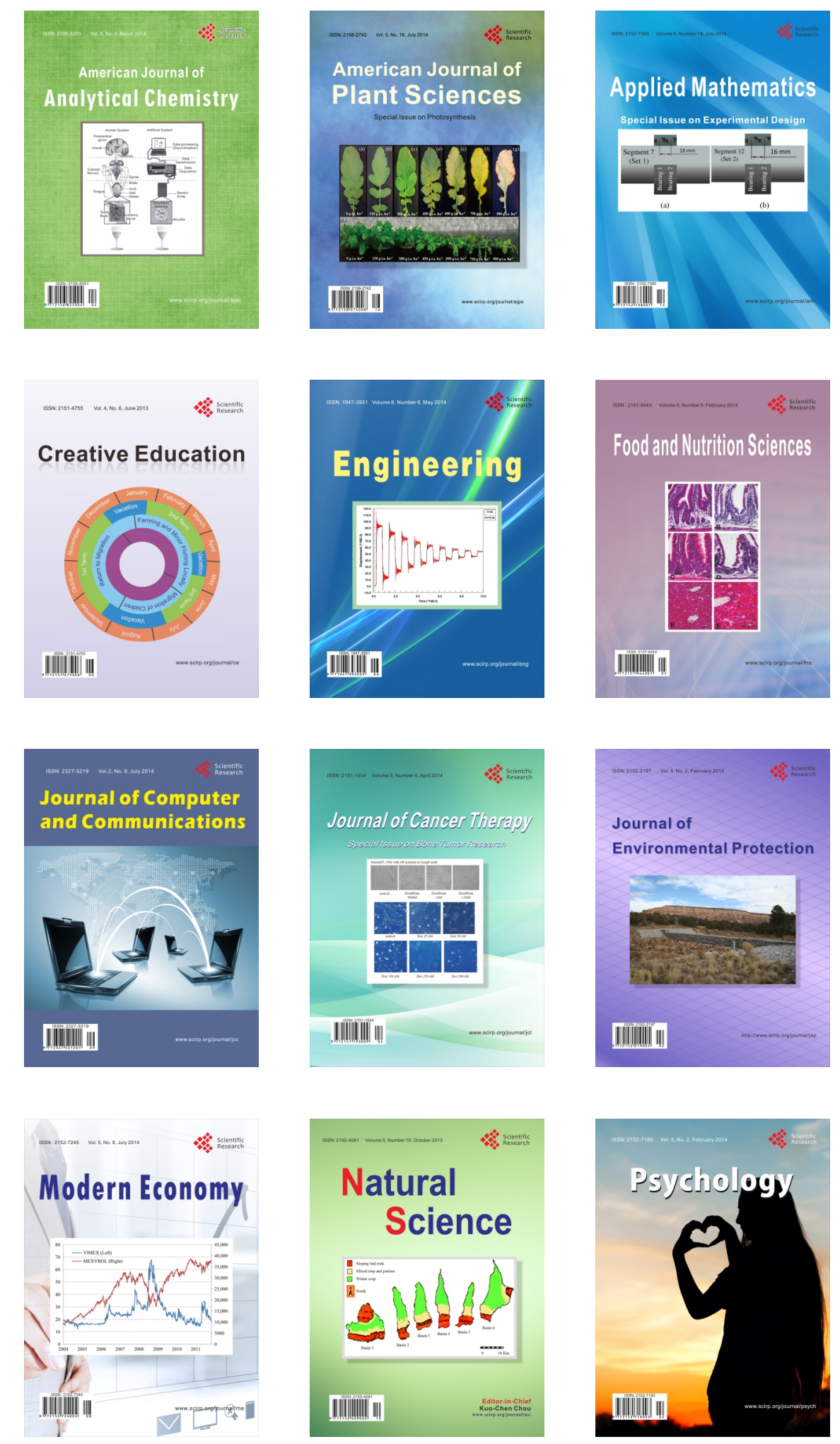